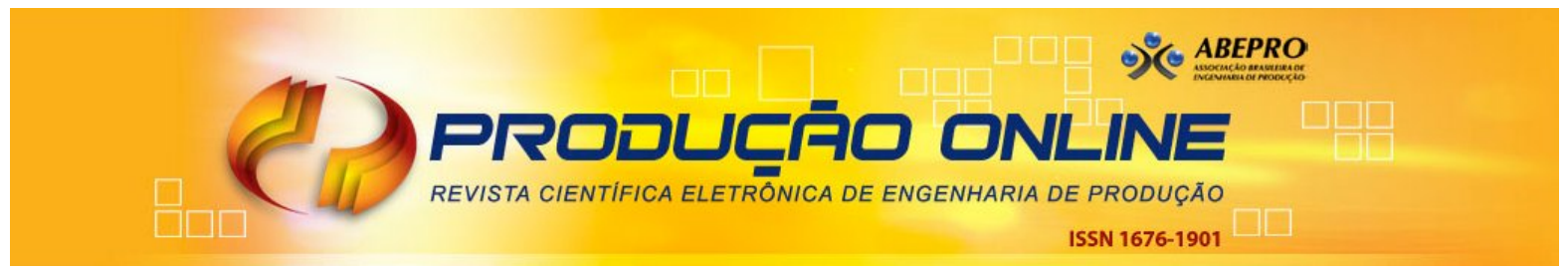

\title{
PREVISÃO DO VOLUME EXPORTADO PARA A FRUTICULTURA BRASILEIRA VIA ANÁLISE DE SÉRIES TEMPORAIS: UMA ABORDAGEM ARIMA/GARCH
}

\section{FORECASTING OF EXPORTED VOLUME FOR BRAZILIAN FRUITS BY TIME SERIES ANALYSIS: AN ARIMA/GARCH APPROACH}

\author{
Abdinardo Moreira Barreto de Oliveira*E-mail: abdinardo@yahoo.com.br \\ Antônio Pires Crisóstomo* E-mail: antonio.crisostomo@univasf.edu.br \\ *Universidade Federal do Vale do São Francisco (UNIVASF), Juazeiro, BA
}

\begin{abstract}
Resumo: O objetivo deste trabalho foi propor modelos de previsão econométricos para o volume exportado da fruticultura brasileira, com vistas a auxiliar o planejamento e controle de sua produção, motivado também pela constatação de poucos estudos publicados tratando desse tema. Nesse sentido, empregou-se os modelos ARIMA/GARCH, considerando-se, outrossim, a ocorrência de uma sazonalidade estocástica multiplicativa nessas séries históricas. Foram coletadas 300 observações de peso líquido (kg) exportado entre jan/1989 a dez/2013 das seguintes frutas: abacaxi, banana, laranja, limão-lima, maçã, mamão, manga, melancia, melão e uva, cujo critério de seleção foi a sua importância na pauta de exportação frutícola, pois representavam $97 \%$ do total de dólares recebidos e $99 \%$ do volume vendido em 2010, de uma população de 28 tipos de frutas exportadas. Os resultados indicam que só não foi observada a existência de sazonalidade multiplicativa de 12 meses na banana e na manga. Por outro lado, foram identificadas dois grupos de frutas: (1) as que são exportadas continuamente, e (2) as que possuem picos de exportação. Sobre a qualidade dos modelos de previsão, eles foram considerados satisfatórios para seis das dez frutas analisadas. Quanto à volatilidade, verificou-se uma alta persistência das séries da banana e do mamão, apontando para a existência de uma quebra estrutural na série de dados, que pode estar associada às crises econômicas ocorridas nos últimos 17 anos.
\end{abstract}

Palavras-chave: Fruticultura exportadora. Previsão. Séries Temporais. Modelos ARIMA. Modelos GARCH.

\begin{abstract}
The aim of this paper was to offer econometric forecasting models to the Brazilian exported volume fruits, with a view to assisting the planning and production control, also motivated by the existence of a few published papers dealing with this issue. In this sense, it was used the ARIMA/GARCH models, considering, likewise, the occurrence of a multiplicative stochastic seasonality in these series. They were collected 300 observations of exported net weight $(\mathrm{kg})$ between Jan/1989 and Dec/2013 of the following fruits: pineapple, banana, orange, lemon, apple, papaya, mango, watermelon, melon and grape, which selection criteria was its importance in the exported basket fruit, because they represented $97 \%$ of total received dollars, and $99 \%$ of total volume sold in 2010, of a population about 28 kinds of exported fruits. The results showed that it was not only observed the existence of a 12 month multiplicative seasonality in banana and mango. On the other hand, they were identified two fruits groups: (1) those which are continuously exported, and (2) those which have export peaks. On the quality of the models, they were considered satisfactory for six of the ten fruits analyzed. On the volatility, it was seen a high persistence in banana and papaya series, pointing to the existence of a structural break in time series, which could be linked to the economic crises happened in the last 17 years.
\end{abstract}

Keywords: Exported fruits. Forecasting. Time series. ARIMA models. GARCH models. 


\section{INTRODUÇÃO}

Mundialmente, o setor alimentício tem tido uma tendência de crescimento na produção e no consumo de produtos naturais, especialmente as frutas. Dentre outros aspectos, Bueno e Baccarin (2012) mostraram que o valor das exportações de frutas frescas brasileiras apresentou, entre 1997 e 2008, uma taxa de crescimento de 105\%, frente a uma retomada das importações (aumento de 59\%), o que contribuiu para o incremento positivo de $112 \%$ nessa balança comercial.

Ainda falando sobre os players internacionais desse mercado, os Estados Unidos merecem destaque, por ser um importador líquido. Entre 2003 e 2006, as importações norte-americanas de frutas frescas e de vegetais cresceram de US\$2,7 bilhões para US $\$ 7,9$ bilhões, representando cerca de $44 \%$ no consumo desses produtos. Ademais, o Brasil está inserido numa das poucas regiões que conseguem transacionar com os EUA - os países do hemisfério sul - os quais forneceram cerca de $32 \%$ das frutas frescas importadas (NZAKU; HOUSTON; FONSAH, 2010).

Independente do cenário favorável à pauta do comércio internacional frutícola, a previsão de elementos-chave do processo produtivo empresarial sempre é necessária, considerando os custos e dilemas envolvidos nas decisões sobre o que, quanto e como produzir, de maneira a equilibrar a oferta (o comportamento dos produtores) e a demanda (o comportamento dos consumidores) em questão (PINDYCK; RUBINFELD, 2013). Neste quesito, é fundamental conceber os riscos inerentes dessa atividade primária, notadamente aqueles ligados às variações nos níveis de estoque, produção e concentração das exportações (BALCOMBE, 2010).

Portanto, as previsões - por serem os valores 'mais prováveis' das melhores estimativas disponíveis sobre o futuro - são imprescindíveis para o planejamento e a estratégia de qualquer organização, especialmente se elas considerarem em seus modelos que tendências passadas podem mudar, e que eventos inesperados, raramente registrados anteriormente, podem voltar a ocorrer (MAKRIDAKIS, 1996).

E em se tratando desses temas, a previsão de demanda/vendas é um ativo muito importante e valioso para as empresas, uma vez que várias de suas atividades e decisões internas são abalizadas em previsões, como o planejamento e controle da produção, as análises financeiras e o lançamento de novos produtos, dentre outras (DANESE; KALCHSCHMIDT, 2011a, 2011b). 
Particularmente sobre o mercado frutícola brasileiro, é comum encontrar trabalhos econométricos que focam no comportamento e previsão de preços (OLIVEIRA; MELO SOBRINHO, 2009; OLIVEIRA, 2010; LIMA; CARMO; MEGLIORINI, 2012; LIMA; SILVA; SANTOS, 2013), mas são raros os que tratam do comportamento e previsão de sua demanda (SOUZA et al., 2013). Isto dificulta o estudo de certas características que podem surgir em séries temporais, como tendências, sazonalidade, pontos influentes (atípicos), heteroscedasticidade condicional e não-linearidade (MORETTIN; TOLOI, 2006; MORETTIN, 2011). Ainda que tais aspectos sejam comuns em séries econômicas e financeiras, eles podem surgir em séries que envolvam a demanda/venda de produtos, merecendo assim a atenção dos participantes envolvidos no planejamento empresarial nesse quesito.

Dada esta lacuna acadêmico-empírica, o objetivo deste trabalho é propor equações econométricas de previsão para o volume exportado da fruticultura brasileira, com vistas a evidenciar as características em séries temporais supracitadas, cujos resultados poderiam auxiliar o planejamento e controle de sua produção. Ademais, é importante mencionar que o uso de técnicas de previsão, a adoção de abordagens decisórias baseadas em previsões e o uso de várias fontes de informação são todas positivamente relacionadas com 0 desempenho empresarial nos custos e na distribuição (DANESE; KALCHSCHMIDT, 2011a).

Aliado a este fato, também se deve considerar as especificidades de frutas, particularmente a sensibilidade à sazonalidade da oferta e da demanda, perecibilidade e acentuada diversidade, o que deixa mais latente a necessidade de utilização de um adequado método de previsão de demanda. Nesse sentido, é sugerida a Análise de Séries Temporais, particularmente o emprego dos modelos ARIMA e GARCH, descritos a seguir.

\section{OS MODELOS ARIMA E GARCH}

A Análise de Séries Temporais é um método quantitativo de previsão que realiza a projeção de valores futuros de uma variável, fundamentada eminentemente em suas observações passadas, organizadas de forma sequencial e em intervalos de tempo específicos escolhidos pelo analista. Assim o modelo econométrico construído para a previsão da série temporal permite que os dados analisados 
"falem por si", sem recorrer a uma teoria subjacente específica para possibilitar sua interpretação (BOX; JENKINS; REINSEL, 1994).

Dentre os métodos econométricos de previsão disponíveis, o modelo Autorregressivo Integrado e de Médias Móveis (ARIMA) anunciou uma nova geração de ferramentas de previsão de séries temporais, dada a combinação de três filtros para a estimação dos valores futuros: o autorregressivo (AR), o de integração (I) e o de médias móveis (MA).

Os modelos $A R(p)$ acontecem quando se verifica a presença de correlação entre os $p$ valores observados na série temporal. Já os modelos $\mathrm{MA}(q)$ pesquisam a estrutura de autocorrelação dos resíduos de previsão, que é examinada sempre que existir uma correlação entre a média móvel dos $q$ termos de erro sucessivo na série temporal. Caso a série apresente ambas as características, os modelos podem ser combinados, criando um processo $\operatorname{ARMA}(p, q)$. Por fim, o filtro I(d) é usado quando se observa que a série temporal não é estacionária, ou seja, é integrada. Portanto, após calcular a diferença entre os valores subjacentes da série $d$ vezes, é possível torná-la estacionária, oferecendo assim uma base válida para a previsão.

O Quadro 1 mostra as equações do modelo ARIMA (p, d, q), onde $Y_{t}^{*}$ é o valor observado (com defasagem $d$ caso seja não estacionário), $\varphi$ t é o coeficiente autorregressivo, $\theta_{\mathrm{t}}$ é o coeficiente de média móvel, $\mu$ é uma constante, $\varepsilon \mathrm{t}$ é o termo de erro estocástico de ruído branco, e $B$ é o operador de defasagem, onde $B^{n} Y_{t}^{*}=$ $Y^{*} t-n$.

Quadro 1 - Equações de previsão para o modelo ARIMA

\begin{tabular}{|c|l|}
\hline Método & Equação \\
\hline $\operatorname{AR}(p)$ & $Y_{\mathrm{t}}^{*}=\varphi_{1} Y_{\mathrm{t}-1}^{*}+\cdots+\varphi_{\mathrm{p}} Y_{\mathrm{t}-\mathrm{p}}^{*}+\varepsilon_{\mathrm{t}} \equiv \varphi_{\mathrm{p}}(B) Y_{\mathrm{t}}^{*}=\left(1-\varphi_{1} B-\cdots-\varphi_{\mathrm{p}} B^{p}\right) Y_{\mathrm{t}}^{*}=\varepsilon_{\mathrm{t}}$ \\
$\operatorname{MA}(\mathrm{q})$ & $Y_{\mathrm{t}}^{*}=\mu-\theta_{1} \epsilon_{\mathrm{t}-1}-\cdots-\theta_{\mathrm{q}} \epsilon_{\mathrm{t}-\mathrm{q}}+\varepsilon_{\mathrm{t}} \equiv \theta_{\mathrm{q}}(B) \varepsilon_{\mathrm{t}}=\left(1-\theta_{1} B-\cdots-\theta_{\mathrm{q}} B^{q}\right) \varepsilon_{\mathrm{t}}=Y_{\mathrm{t}}^{*}$ \\
$\operatorname{ARMA}(\mathrm{p}, \mathrm{q})$ & $Y_{\mathrm{t}}^{*}=\varphi_{1} Y_{\mathrm{t}-1}^{*}+\cdots+\varphi_{p} Y_{\mathrm{t}-\mathrm{p}}^{*}-\theta_{1} \varepsilon_{\mathrm{t}-1}-\cdots-\theta_{\mathrm{q}} \varepsilon_{\mathrm{t}-\mathrm{q}}+\varepsilon_{\mathrm{t}} \equiv \varphi_{\mathrm{p}}(B) Y_{\mathrm{t}}^{*}=\theta_{\mathrm{q}}(B) \varepsilon_{\mathrm{t}}$
\end{tabular}

Fonte: Box, Jenkins e Reinsel (1994), Morettin e Toloi (2006), e Morettin (2011)

Todavia, é possível que ao longo da série temporal apareça o efeito da sazonalidade estocástica, isto é, a ocorrência de fenômenos numa periodicidade regular $S$, mas cujo componente sazonal varia conforme o tempo. Nesses casos, é sugerido o uso de um termo sazonal multiplicativo (SARIMA) do tipo $(p, d, q) \times(P, D$, $\mathrm{Q})_{s}$, onde $\mathrm{P}$ indica a ordem do operador autorregressivo sazonal; D indica o número 
de diferenças sazonais; Q indica a ordem do operador de médias móveis sazonal; e $S$ representa o período da sazonalidade. As equações que representam o modelo SARIMA, na sua forma polinomial reduzida B são (BOX; JENKINS; REINSEL, 1994; MORETTIN; TOLOI, 2006; MORETTIN, 2011):

$$
\begin{gathered}
\varphi_{p}(B) \Phi_{p}\left(B^{s}\right) \nabla^{d} \nabla_{s}^{D} Y_{t}^{*}=\theta_{q}(B) \Theta_{Q}\left(B^{S}\right) \varepsilon_{t} \\
\underbrace{\left(1-\varphi_{1} B-\cdots-\varphi_{p} B^{p}\right)}_{A R(p)} \underbrace{\left(1-\Phi_{1} B^{S}-\cdots-\Phi_{p} B^{P S}\right)}_{\text {AR(p)Sazonal }} \underbrace{(1-B)^{d}}_{\text {Diferença }} \underbrace{\left(1-B^{S}\right)^{D}}_{\text {Dif. Sazonal }} Y_{t}^{*}= \\
\underbrace{\left(1-\theta_{1} B-\cdots-\theta_{q} B^{q}\right)}_{\text {MA(q) Sazonal }} \underbrace{\left(1-\Theta_{1} B^{S}-\cdots-\Theta_{Q} B^{Q S}\right)} \varepsilon_{t}
\end{gathered}
$$

Onde $\varphi_{p}(B)$ representa os termos autorregressivos de ordem $p A R(p) ; \Phi_{P}\left(B^{s}\right)$ indica o termo autorregressivo sazonal $s$ de ordem $\mathrm{P} ; \nabla^{\mathrm{d}}$ é o operador de diferenças simples; $\nabla_{\mathrm{s}}^{\mathrm{D}}$ é o operador de diferenças sazonais, $Y_{t}^{*}$ representa a série histórica analisada; $\theta_{\mathrm{q}}(\mathrm{B})$ indica os termos de médias móveis de ordem q $\mathrm{MA}(\mathrm{q}) ; \Theta_{\mathrm{Q}}\left(\mathrm{B}^{\mathrm{s}}\right)$ é o termo de média móvel sazonal $S$ de ordem Q; $\varepsilon$ t é o termo de erro aleatório.

Contudo, um aspecto que precisa ser acatado no uso dos modelos ARIMA é a constância de sua variância, isto é, seus valores futuros não dependerem de seus valores passados. Quando esta condição não é sustentada, uma correção para a sua estimação deve ser considerada, sob pena de se ter suas previsões enviesadas. Neste quesito, Engle $(1982,1983)$ propôs uma nova classe de modelos estocásticos que admitem a variância mudar temporalmente em função dos erros de previsão passados, o qual chamou de heterocedasticidade condicional autorregressiva $[\mathrm{ARCH}(\mathrm{q})]$. Assim, a variância condicionada $\left(\sigma^{2} \mathrm{t}\right)$ passou a ser estimada a partir da seguinte expressão:

$$
\sigma_{t}^{2}=\alpha_{0}+\sum_{i=1}^{q} \alpha_{i} \varepsilon_{t-i}^{2}
$$

Onde $\alpha_{0}$ e $\alpha_{i}$ são coeficientes não-negativos a serem estimados, cuja soma deve ser menor que um, e $\varepsilon^{2}$ i indica os erros de previsão passados ao quadrado. Dado que a variância é uma medida de risco academicamente aceita, foi possível então observar essa mudança de risco ao longo do tempo a partir das inovações ocorridas no passado, chamada de volatilidade. Sobre ela, Bera e Higgins (1993) explicam que a equação (6) intenta em reproduzir os agrupamentos de grandes 
choques nela presentes, bem como comentam que o valor de $q$ estabelece a extensão do tempo no qual o choque persiste na variância condicional: quanto maior seu valor for, mais longo será o evento da volatilidade.

Entretanto, caso os valores de $q$ sejam grandes, isto demonstra a existência de uma volatilidade de memória longa. Logo, Bollerslev (1986) alertou que tal fenômeno pode causar a violação da não-negatividade dos coeficientes alfas (o que implica em variâncias negativas) e a perda de parcimônia da equação (6), cujas ocorrências foram observadas em vários estudos empíricos até então (ENGLE, 1982, 1983). Nesse sentido, Bollerslev (1986) apresentou uma extensão da classe dos modelos ARCH que lidam com a situação de memória longa na volatilidade, mas com uma estrutura de valores defasados mais flexível, o qual chamou de heterocedasticidade condicional autorregressiva generalizada [GARCH(p,q)]:

$$
\sigma_{t}^{2}=\alpha_{0}+\sum_{i=1}^{q} \alpha_{i} \varepsilon_{t-i}^{2}+\sum_{i=1}^{p} \beta_{i} \sigma_{t-i}^{2}
$$

Onde $\beta_{i}$ são os coeficientes não-negativos a serem estimados para as variâncias condicionadas passadas, $p$ indica o tamanho do tempo no qual a variância condicional passada continua na atual, e o somatório dos coeficientes alfas e betas devem ser menor do que um para que a variância condicionada seja positiva e fracamente estacionária. Se $p=0$, a equação (7) torna-se a equação (6), revelando ser essa última um caso específico. Posto isso, Bera e Higgins (1993) comentam que a generalização do $\operatorname{ARCH}(q)$ para o $\operatorname{GARCH}(p, q)$ é similar à generalização do processo $M A(q)$ para o $\operatorname{ARMA}(p, q)$, cuja intenção (do $G A R C H$ ) é parcimoniosamente sumarizar altas ordens $q$ de um processo ARCH.

Todavia, Engle e Bollerslev (1986) perceberam depois que se as funções de autocorrelação e autocorrelação parcial dos erros ao quadrado passados diminuírem de forma relativamente lenta, a soma dos coeficientes alfas e betas que melhor se ajustam ao modelo fica muito próxima de um $(\alpha+\beta \cong 1)$, indicando uma elevada persistência na variância, na qual a informação atual continua importante para a previsão das variâncias condicionais para todos os horizontes de tempo, evitando o decaimento exponencial dos pesos para longas defasagens. Em outras palavras, os choques na variância não diminuem ao longo do tempo, mas sim se fortalecem. Nesse caso, o processo é chamado de integrado na variância, ou IGARCH, uma vez 
que a variância incondicional ( $\left.\alpha_{0}\right)$ não existe, e a série histórica da variância não é estacionária, tal como num processo estocástico de passeio aleatório.

Por outro lado, o revés do modelo IGARCH está na ausência de qualquer motivação teórica, a qual poderia conduzir a uma má especificação dos parâmetros alfas e betas. Tendo isso em mente, Lamoureux e Lastrapes (1990) confirmaram a hipótese de que a aparente alta persistência na variância é devido à variação dos parâmetros GARCH ao longo do tempo, ou seja, ocorre uma mudança (ou quebra) estrutural na variância incondicional do processo estocástico, sugerindo a existência de, pelo menos, dois modelos GARCH explicativos.

\section{METODOLOGIA}

Realizou-se um levantamento na população de frutas exportadas brasileiras, escolhendo-se os seguintes produtos para a amostra de investigação: abacaxi, banana, laranja, limão-lima, maçã, mamão, manga, melancia, melão e uva, totalizando 10 produtos de uma população de 28 tipos de frutas exportadas em 2010. O critério de escolha desses produtos foi a sua importância na pauta de exportação frutícola, pois representavam $97 \%$ do total em dólares recebidos e 99\% do volume vendido (IBRAF, 2011; BUENO; BACCARIN, 2012).

Os dados referentes às frutas selecionadas foram coletados no banco de dados AliceWeb2 (BRASIL, 2014), do Ministério do Desenvolvimento, Indústria e Comércio Exterior (MDIC), conforme codificações mostradas no Quadro 2. Nele, é possível perceber que o mesmo produto apresenta mais de uma codificação e mais de um código AliceWeb2, ou produtos anteriormente separados foram agregados num mesmo código. Isto ocorreu porque entre 1989 e 1996 o sistema de codificação adotado pelo MDIC foi o NBM; somente a partir de 1997 que o NCM substituiu a codificação anterior, permanecendo até hoje.

Além disso, é notório que na mesma codificação, um produto podia ter mais de um código AliceWeb2, como foram os casos da banana, do limão-lima, e da manga. Esta última merece uma atenção especial, porque entre 1989 e 1996, ela tinha código único, independentemente de ser fresca ou seca; contudo, a partir de Jan/1997, todas as informações sobre suas exportações foram agregadas aos da goiaba e dos mangostões, voltando a possui código único somente a partir de Ago/2003. 
Para os fins do artigo, todas as situações mostradas no Quadro 2 foram consideradas como se pertencessem a uma única série temporal, e quando da ocorrência de dados concomitantes, estes foram agregados num único dado no tempo. Portanto, entre Jan/1989 e Dez/2013, foram coletadas 300 observações mensais do peso líquido $(\mathrm{kg})$ por produto listado.

Quadro 2 - Codificação das frutas segundo o AliceWeb2

\begin{tabular}{|c|c|c|c|}
\hline Produto & Tipo & Codificação & $\begin{array}{c}\text { Código } \\
\text { AliceWeb2 }\end{array}$ \\
\hline \multirow{2}{*}{ Abacaxi } & (Ananás) Fresco ou seco & NBM & 0804300000 \\
\hline & Fresco ou seco & NCM & 08043000 \\
\hline \multirow{5}{*}{ Banana } & Fresca & NBM & 0803000100 \\
\hline & Seca & NBM & 0803000200 \\
\hline & Fresca ou seca & NCM & 08030000 \\
\hline & Da terra, fresca ou seca & $\mathrm{NCM}$ & 08031000 \\
\hline & Fresca ou seca, sem bananas-da-terra & NCM & 08039000 \\
\hline \multirow{2}{*}{ Laranja } & Fresca ou seca & NBM & 0805100000 \\
\hline & Fresca ou seca & $\mathrm{NCM}$ & 08051000 \\
\hline \multirow{3}{*}{ Limão e lima } & Limão, fresco ou seco & NBM & 0805300100 \\
\hline & Lima, fresco ou seco & NBM & 0805300200 \\
\hline & Limão e lima, fresco ou seco & NCM & 08053000 \\
\hline \multirow{2}{*}{ Maçã } & Fresca & NBM & 0808100000 \\
\hline & Fresca & NCM & 08081000 \\
\hline \multirow{2}{*}{ Mamão } & (Papaia) Fresco & NBM & 0807200000 \\
\hline & (Papaia) Fresco & NCM & 08072000 \\
\hline \multirow{3}{*}{ Manga } & Fresca ou seca & NBM & 0804500200 \\
\hline & $\begin{array}{c}\text { Goiabas, mangas e mangostões, frescos e } \\
\text { secos }\end{array}$ & NCM & 08045000 \\
\hline & Fresca ou seca & NCM & 08045020 \\
\hline \multirow{2}{*}{ Melancia } & Fresca & NBM & 0807100200 \\
\hline & Fresca & NCM & 08071100 \\
\hline \multirow{2}{*}{ Melão } & Fresco & NBM & 0807100100 \\
\hline & Fresco & NCM & 08071900 \\
\hline \multirow{2}{*}{ Uva } & Fresca & NBM & 0806100000 \\
\hline & Fresca & $\mathrm{NCM}$ & 08061000 \\
\hline
\end{tabular}

Fonte: Elaboração própria, a partir do AliceWeb2 (BRASIL, 2014)

Em caso de ocorrência de dados perdidos (missing values), estes foram preenchidos pela média aritmética dos valores dos períodos adjacentes, de modo a permitir o correto uso dos modelos econométricos acima descritos, bem como preservar quaisquer efeitos de sazonalidade presentes na série histórica.

Os dados foram divididos em dois grupos: o primeiro, nomeado dentro-daamostra, contém 264 observações (jan-1989 a dez-2010) e foi utilizado para construir os modelos econométricos de previsão; o segundo, nomeado fora-da- 
amostra, contém 36 observações (jan-2011 a dez-2013), foi empregado para testar a capacidade preditiva desses modelos.

Sobre o correto uso dos modelos ARIMA/GARCH, é preciso atender a quatro etapas. Inicialmente, foram testadas as hipóteses nulas de existência de raiz unitária e de estacionariedade nas séries de peso líquido $(\mathrm{kg})$, respectivamente através dos testes Dickey-Fuller Aumentado (ADF) (DICKEY; FULLER, 1979; SAID; DICKEY, 1984) e KPSS (KWIATKOWSKI et al., 1992), conforme orientação de Brooks (2008). Caso as séries de peso líquido $(\mathrm{kg})$ se comportem como um passeio aleatório (aceita $\mathrm{H}_{0}$ em ADF ou rejeita $\mathrm{H}_{0}$ em KPSS), foram utilizadas em seu lugar as séries de $\log$ diferença/retorno $\left[\ln \left(V_{n} V_{n-1}\right)\right]$, que tendem a se comportarem como um ruído branco (rejeita $\mathrm{H}_{0}$ em ADF e aceita $\mathrm{H}_{0}$ em KPSS).

Atendendo aos requisitos de ausência de raiz unitária e de estacionariedade, a quantidade de termos $\operatorname{AR}(p), M A(q), S A R(P)$ e $S M A(Q)$ presentes na equação foi definida pelo menor Critério de Informação Bayesiano de Schwarz (1978) [SBIC], assim calculado (BOX; JENKINS; REINSEL, 1994; BROOKS, 2008):

$$
\begin{aligned}
& \text { Max } L=-\frac{N}{2}\left[\ln (2 \pi)+\ln \left(\sigma^{2}\right)\right]-\sum_{n=1}^{N} \frac{\varepsilon_{n}^{2}}{2 \sigma^{2}} \\
& \text { Min SBIC }=-2\left(\frac{L}{N}\right)+\frac{k \ln (N)}{N}
\end{aligned}
$$

Onde $k$ é o número de termos, $\mathrm{N}$ é o tamanho da amostra, $\sigma^{2}$ é a variância estimada, e $\varepsilon \mathrm{t}^{2}$ são os resíduos estimados da equação. Logo, os coeficientes dos termos da equação foram calculados pela maximização de sua função de logverossimilhanda $L$ (eq. 8), e o modelo escolhido foi aquele que teve o menor SBIC (eq. 9). Nesse sentido, foram testados modelos ARMA (p, q), com p, $q \in[1,10]$, e modelos SARMA $(p, q) \times(P, Q)$, com $p, q \in[1,5] ; P, Q \in[6 ; 12]$. Os termos selecionados tem significância estatística de 5\% via distribuição $t$ de Student, com N - $k$ graus de liberdade. O SBIC foi escolhido para a seleção das equações econométricas porque ele é mais sensível ao acréscimo de novos termos (o modelo é penalizado devido à perda de graus de liberdade), tendendo a mantê-las parcimoniosas e simples, ainda que nenhum critério de informação seja considerado definitivamente superior (BOX; JENKINS; REINSEL, 1994; BROOKS, 2008).

Depois foram feitos os diagnósticos para os modelos estimados, verificandose (I) a ausência de correlação serial e (II) a presença de homocedasticidade na 
variância. Sobre o primeiro diagnóstico, se for detectada a presença de correlação serial, o modelo foi revisado até ela ter sido eliminada, mesmo que seu SBIC seja o menor valor encontrado.

Nesse quesito, foram usados os testes $\mathrm{Q}_{(\mathrm{m})}$ de Ljung e Box (1978) [LB(m)], com distribuição $\chi^{2}$ e $(m-p-q)$ graus de liberdade, e o de Breusch (1978) e Godfrey (1978) via multiplicador de Lagrange [BG-LM(m)], também com distribuição $\chi^{2}$ e $m$ graus de liberdade, sob a hipótese nula $\left(\mathrm{H}_{0}\right)$ de ausência de correlação serial entre os resíduos, sendo $m$ o $\mathrm{n}^{0}$ de defasagens e igual a 10. Caso $\chi^{2}$ calculado $>\chi^{2}$ crítico, rejeita-se a hipótese de ausência de correlação serial nos resíduos; do contrário, ela é aceita, com significância de $1 \%$.

Sobre o segundo diagnóstico, Engle (1982) apresentou um procedimento consistente de estimação de heterocedasticidade via multiplicador de Lagrange, obtido através da regressão da seguinte equação por mínimos quadrados ordinários:

$$
\varepsilon_{t}^{2}=\alpha_{0}+\sum_{i=1}^{m} \alpha_{i} \varepsilon_{t-i}^{2}+v_{t} \quad(10) \quad \text { ARCH LM }=\mathrm{NR}_{\varepsilon}^{2}
$$

Onde $\varepsilon$ t são os resíduos obtidos da matriz original de regressores $A R M A(p$, $\mathrm{q}) / \operatorname{SARMA}(\mathrm{p}, \mathrm{q}) \times(\mathrm{P}, \mathrm{Q}), \mathrm{N}$ é o tamanho da amostra e $\mathrm{R}^{2}$ é o coeficiente de determinação da equação (10). O teste $\mathrm{ARCH}-\mathrm{LM}(\mathrm{m})$ tem distribuição $\chi^{2} \mathrm{com} m$ graus de liberdade, sendo $m$ igual a um. Se $\chi^{2}$ calculado $<\chi^{2}$ crítico, existe homocedasticidade na variância, com significância de 1\%. Em caso de heterocedasticidade na variância, aplicou-se os modelos da família GARCH, cuja estimação dos parâmetros em conjunto com os do ARIMA se deu pela maximização da equação (8), com $\sigma^{2}$ representando as equações (6) e (7) conforme foi o modelo construído.

A qualidade de previsão das equações construídas foi testada pela estatística $U_{2}$ de Theil (BLIEMEL, 1973), após a estimação do peso líquido (kg) mensal:

$$
U_{2}=\sqrt{\frac{\sum_{i=1}^{N}\left(P_{i}-A_{i}\right)^{2}}{\sum_{i=1}^{N} A_{i}^{2}}}
$$

Onde $\mathrm{P}_{\mathrm{i}}$ e $\mathrm{A}_{\mathrm{i}}$ são, respectivamente, os valores previstos e atuais, e $\mathrm{N}$ indica o tamanho da amostra, que foi igual às 300 observações. Quanto mais próximo de zero for, melhor será a qualidade de previsão do modelo. Os estimadores ARIMA, GARCH [com $\varepsilon^{2} \sim$ Distribuição Normal] e $U_{2}$ foram obtidos via software EViews ${ }^{\odot}$ 8.0. 


\section{RESULTADOS}

O apêndice A traz os gráficos referentes ao comportamento do volume exportado mensal das frutas entre janeiro-1989 e dezembro-2013, enquanto que o apêndice B mostra os mesmos resultados no formato sazonal, ambos em kg. Neles, é possível verificar, a priori, algumas características das séries temporais, a saber:

1. Em relação à tendência, três frutas apresentam diminuição no volume exportado (abacaxi, banana e laranja), enquanto que as demais frutas apresentam ou estabilidade ou aumento no volume exportado;

2. A respeito da sazonalidade, somente duas frutas (banana e mamão) não apresentaram tal característica. Quanto às demais, somente a maçã mostrou este resultado para $01^{\circ}$ semestre, enquanto que para as frutas restantes, os picos de sazonalidade ocorreram no $2^{\circ}$ semestre;

3. Sobre os pontos atípicos, todas as frutas apresentaram, pelo menos, uma vez este evento, marcado como os máximos e mínimos de volume exportado. Complementarmente a isso, também é mostrado um histograma no apêndice $A$, indicando onde está a concentração dos volumes exportados mais frequentes;

Focalizando agora na econometria dessas séries temporais, a Tabela 1 mostra os resultados para os testes das hipóteses nulas de existência de raiz unitária e de estacionariedade nas séries de volume exportado das frutas brasileiras.

Tabela 1 - Resultados dos testes de raiz unitária e estacionariedade

\begin{tabular}{lcccc}
\hline \multirow{2}{*}{ Produto (N = 264) } & \multicolumn{2}{c}{ Série Volume } & \multicolumn{2}{c}{ Série dlog(Volume) } \\
\cline { 2 - 5 } Abacaxi & ADF & KPSS & ADF & KPSS \\
Banana & $-1,4850$ & 0,0942 & $-5,3805^{*}$ & 0,0341 \\
Laranja & $-2,8954$ & 0,2103 & $-21,7387^{*}$ & 0,0685 \\
Limão/Lima & $-4,4386^{*}$ & 0,0445 & $-12,6293^{*}$ & 0,0581 \\
Maçã & $-1,8145$ & $\mathbf{0 , 5 5 6 9 *}$ & $-5,5904^{*}$ & 0,0786 \\
Mamão & $-3,5057$ & 0,0519 & $-12,4571^{*}$ & 0,0410 \\
Manga & $-0,8044$ & $\mathbf{0 , 2 5 1 3}$ & $-\mathbf{9 , 9 3 7 0 ^ { * }}$ & 0,1269 \\
Melancia & $-1,9965$ & 0,1330 & $-11,5226^{*}$ & 0,1171 \\
Melão & $-1,8254$ & 0,1063 & $-14,4004^{*}$ & 0,0445 \\
Uva & $-1,6290$ & 0,1859 & $-17,8986^{*}$ & 0,0238 \\
Valores Críticos (1\%) & $-2,0461$ & $\mathbf{0 , 2 4 3 6}$ & $-22,5661^{*}$ & 0,0458 \\
* Significativo a 1\%. & & & &
\end{tabular}

Revista Produção Online, Florianópolis, SC, v.15, n. 2, p. 553-572, abr./jun. 2015 
Fonte: Elaboração própria

Num primeiro momento, percebe-se que somente a série de dados da laranja atende aos requisitos de ausência de raiz unitária e de estacionariedade; nas demais, a existência de raiz unitária foi detectada, bem como particularmente a não estacionariedade foi vista nas séries de dados do limão/lima, do mamão e da uva, o que implicou no uso de séries de log diferença/retorno, com $d=1$.

Ao reaplicar os testes ADF (com intercepto e tendência) e KPSS nas séries de log diferença/retorno, foi detectada tanto a ausência de raiz unitária como a presença de estacionariedade em todas elas, tornando-as assim aptas para a aplicação dos modelos ARIMA/GARCH. É importante dizer que não se empregou nas análises posteriores a log diferença/retorno na série de dados da laranja, dado que ela já atendia a esses requisitos.

Uma vez que as séries de volume exportado serão aqui modeladas a partir de suas séries de retornos, é importante comentar que estas, além de serem estacionárias (movem-se no tempo aleatoriamente em torno de uma média uniforme), também podem ser interpretadas a partir da hipótese da ergodicidade (HERSCOVICI, 2005), cujo futuro pode ser previsto por cálculos probabilísticos, que apontará as possíveis direções dos componentes desse sistema dinâmico, porém estável, numa evolução previsível que permite a aplicação dos modelos ARIMA.

Quadro 3 - Equações de previsão para as séries de dados das frutas exportadas

\begin{tabular}{|c|c|}
\hline Produto & Equação \\
\hline Abacaxi & $(1+0,8781 B)\left(1-0,9493 B^{12}\right)(1-B)\left(1-B^{12}\right) Y_{t}^{*}=\left(1-0,4499 B+0,4013 B^{2}\right)(1+0,7411 A$ \\
\hline Banana & $\begin{array}{l}\left(1+0,2586 B-0,4435 B^{a}\right)(1-B) Y_{t}^{*}=\left(1+0,4971 B^{a}\right) \varepsilon_{t} \\
\sigma_{t}^{2}=0,1276 \varepsilon_{t-1}^{2}+0,8724 \sigma_{t-1}^{2}\end{array}$ \\
\hline Laranja & $(1-0,3804 B)\left(1-0,9463 B^{12}\right) Y_{t}^{*}=6.562 .760+\left(1+0,7968 B^{12}\right) \varepsilon_{t}$ \\
\hline $\begin{array}{l}\text { Limão/Li } \\
\text { ma }\end{array}$ & $(1-0,5813 B)\left(1-0,8772 B^{12}\right)(1-B)\left(1-B^{12}\right) Y_{t}^{*}=(1+0,9159 B)\left(1+0,6242 B^{12}\right) \varepsilon_{t}$ \\
\hline Maçã & $\begin{array}{l}\left(1-1,1557 B+0,3327 B^{2}+0,1773 B^{4}\right)\left(1-0,2154 B^{12}\right)(1-B)\left(1-B^{12}\right) Y_{t}^{*}=(1+1,6352 B- \\
\left.0,6398 B^{2}\right) \varepsilon_{t}\end{array}$ \\
\hline Mamão & $\begin{array}{l}\left(1-0,9278 B^{12}\right)(1-B) Y_{t}^{*}=(1+0,5614 B)\left(1+0,9592 B^{12}\right) \varepsilon_{t} \\
\sigma_{t}^{2}=0,1248 \varepsilon_{t-1}^{2}+0,8752 \sigma_{t-1}^{2}\end{array}$ \\
\hline Manga & $\begin{array}{l}\left(1-1,4346 B^{2}+0,6051 B^{2}+0,8531 B^{4}-0,1996 B^{5}\right)(1-B) Y_{t}^{*}=\left(1+0,6625 B+1,6771 B^{2}-\right. \\
\left.1,6034 B^{2}-0,7180 B^{4}+0,9012 B^{5}\right) \varepsilon_{t}\end{array}$ \\
\hline Melancia & $(1-0,4402 B)\left(1-0,9596 B^{12}\right)(1-B)\left(1-B^{12}\right) Y_{t}^{*}=(1+0,9933 B)\left(1+0,7013 B^{12}\right) \varepsilon_{t}$ \\
\hline Melão & $\begin{array}{l}(1-0,4129 B)\left(1-0,9913 B^{12}\right)(1-B)\left(1-B^{12}\right) Y_{t}^{*}=(1+0,9928 B)\left(1+0,7093 B^{12}\right) \varepsilon_{t} \\
\sigma_{t}^{2}=0,5239+0,2049 \varepsilon_{t-1}^{2}\end{array}$ \\
\hline Uva & $(1-0,3348 B)\left(1-0,9694 B^{12}\right)(1-B)\left(1-B^{12}\right) Y_{t}^{*}=(1+0,9575 B)\left(1+0,3453 B^{12}\right) \varepsilon_{t}$ \\
\hline
\end{tabular}

Fonte: Elaboração própria

Revista Produção Online, Florianópolis, SC, v.15, n. 2, p. 553-572, abr./jun. 2015 
Após testar várias combinações de modelos ARIMA (p, q) e SARIMA ( $p$, q) $\times(P, Q)$, com $d=1$, chegou-se aos modelos de previsão das séries de dados, mostrados no Quadro 3. Neles, só não foi observada a existência de sazonalidade multiplicativa de 12 meses na banana e na manga. Por outro lado, foram identificados dois grupos de frutas: (1) as que são exportadas continuamente, e (2) as que possuem picos de exportação. No grupo (1) estão a banana, o limão/lima e o mamão; no grupo (2) estão as demais frutas, nas quais o abacaxi, a laranja, a manga, a melancia, o melão e a uva possuem picos de exportação no $2^{\circ}$ semestre, enquanto que na maçã, tal ocorrência se dá no $1^{\circ}$ semestre (VER APÊNDICE B).

A Tabela 2 mostra o resultado do diagnóstico dos modelos de previsão mostrados no Quadro 3. É importante destacar que os valores do SBIC são os menores encontrados, procurando manter as equações de previsão o mais simples possível, condicionada à ausência de correlação serial de seus resíduos. E a respeito disso, tal ausência foi detectada em todos os modelos pelo teste $\operatorname{LB}_{(10)}$, a 1\% de significância, porém dando-se preferência aos resultados obtidos no teste BG-LM(10), quando disponíveis.

Tabela 2 - Resultados do diagnóstico das equações de previsão

\begin{tabular}{lcccccc}
\hline \multicolumn{1}{c}{ Produto } & $\mathbf{R}^{\mathbf{2}}$ adj. & SBIC & $\mathbf{U}_{\mathbf{2}}$ & $\mathbf{L B}_{(\mathbf{1 0})}$ & BG-LM $_{(\mathbf{1 0})}$ & ARCH-LM $_{(\mathbf{1})}$ \\
\hline Abacaxi & 0,5197 & 1,8903 & 0,3555 & $14,90 \%$ & 12,1887 & 0,8766 \\
Banana & 0,1183 & 0,4231 & 0,1988 & $18,20 \%$ & - & 3,7678 \\
Laranja & 0,7382 & 33,8956 & 0,4315 & $30,20 \%$ & 10,6340 & 5,3565 \\
Limão/Lima & 0,3353 & 2,3114 & 0,2098 & $3,90 \%$ & 13,9199 & 5,0576 \\
Maçã & 0,3460 & 4,5483 & 0,7845 & $12,90 \%$ & 17,5441 & 5,7541 \\
Mamão & 0,3445 & $-0,4498$ & 0,1224 & $8,40 \%$ & - & 3,6564 \\
Manga & 0,5801 & 2,7572 & 0,3497 & $0,20 \%$ & 9,1289 & 4,7522 \\
Melancia & 0,4528 & 3,3411 & 0,2849 & $2,70 \%$ & 12,8212 & 6,1518 \\
Melão & 0,6576 & 2,4834 & 0,2285 & $61,00 \%$ & - & 0,1428 \\
Uva & 0,7390 & 2,8220 & 0,3390 & $1,80 \%$ & 14,1186 & 1,5081 \\
Valores Críticos (1\%) & & & $1 \%$ & 23,2093 & 6,6349 \\
\hline
\end{tabular}

Fonte: Elaboração própria

Sobre a qualidade dos modelos de previsão (o que indica o quão ergódigo a série histórica é), foram identificados três grupos: o primeiro, de alta qualidade $\left(\mathrm{U}_{2} \leq\right.$ 0,33), abrange a banana, o limão/lima, o mamão, a melancia, o melão e a uva; o segundo, de média qualidade $\left(0,33<U_{2} \leq 0,67\right)$, engloba o abacaxi, a laranja e a manga; e o terceiro, de baixa qualidade $\left(U_{2}>0,67\right)$, inclui somente a maçã. 
Os resultados do indicador $U_{2}$ acerca da qualidade de previsão também podem ser reinterpretados a partir da discussão a respeito do impacto das previsões no desempenho das companhias. Ainda que exista na literatura especializada uma defesa contundente sobre o uso isolado de técnicas de previsão porque elas tendem a reduzir os vieses de julgamento e os efeitos das informações irrelevantes (DANESE; KALCHSCHMIDT, 2011a), elas sozinhas não necessariamente melhoram a precisão: existe também nessa mesma literatura uma argumentação de que a eficácia de uma técnica de previsão depende do contexto, com algumas técnicas mais apropriadas do que outras, dadas certas condições do negócio analisado (DANESE; KALCHSCHMIDT, 2011b).

É o que parece estar acontecendo com as frutas que tiveram os resultados de $\mathrm{U}_{2}$ acima de 0,33 (abacaxi, laranja, manga e maçã). Dado que as condições iniciais desses mercados tenham mudado, isso implica na violação da hipótese da ergodicidade (HERSCOVICI, 2005), o que num futuro volátil, as previsões baseadas eminentemente em dados históricos tendem a ser menos eficazes (DANESE; KALCHSCHMIDT, 2011b).

Com vistas a oferecer uma alternativa a essa situação, Kalchschmidt (2012) mostra que de uma perspectiva gerencial, as companhias devem investir na gestão do processo de previsão, o qual é mais abrangente do que o simples uso de técnicas de previsão: consiste também em incorporar aos resultados previstos as informações vindas do mercado (pesquisas de intenção junto aos consumidores, condições econômicas atuais, tendências futuras etc.), transformando tais conjuntos de informações num arcabouço consistente para a tomada de decisões aplicáveis ao contexto interno e externo em que elas se situam, visando obterem melhores desempenhos operacionais de custos e de distribuição.

A respeito da presença de heteroscedasticidade condicional - quarta característica em séries temporais - ela foi encontrada nas séries da banana, do mamão e do melão, indicando assim que a variância da log diferença/retorno dessas séries muda ao longo do tempo. Nesses casos, aplicou-se os modelos GARCH, obtendo-se um modelo $\mathrm{ARCH}(1)$ para o melão, e $\mathrm{GARCH}(1,1)$ integrado para a banana e o mamão, uma vez que a soma de seus coeficientes $\alpha$ e $\beta$ totalizaram um. Ao reaplicar o teste $\mathrm{ARCH}-\mathrm{LM}_{(1)}$, constatou-se a ausência de heterocedasticidade. 
Ademais, é mister enfatizar sobre a ocorrência do GARCH integrado nos resultados deste estudo. Tal situação acontece quando $\alpha_{0}=0$, indicando uma alta persistência na variância decorrente da mudança nos parâmetros GARCH no tempo, os quais afetam o resultado da variância condicional, cujos efeitos dos choques passados na variância corrente ficam mais fortes, apontando para a existência de uma quebra estrutural na série de dados (LAMOUREUX; LASTRAPES, 1990).

Por fim, como uma contribuição adicional aos objetivos desse estudo e de investigações futuras, foi intencionado comentar que, sendo aqui as previsões dos volumes exportados obtidas a partir de suas log diferenças/retornos passados (com exceção da série histórica da laranja), é interessante pontuar que, além delas serem livres de escala, elas são frequentemente utilizadas para se calcular o risco associado à série temporal, a partir da variância desses dados.

Dito isso, poder-se-ia introduzir na análise de séries temporais de retornos de volume exportado um conceito bastante difundido entre os financistas ao se analisar o risco de ativos financeiros, que é o Valor-em-Risco (VaR). Em termos gerais, o VaR "é uma medida da variação potencial máxima do valor de um ativo, sobre um período pré-fixado, com dada probabilidade" (MORETTIN, 2011, p. 194). Assim, as partes interessadas nesse quesito poderiam estimar, por exemplo, a probabilidade de que o log retorno do volume exportado não seja menor que determinado patamar, com previsão de um mês adiante. Assim, se teria uma métrica de perda ligada a um evento extremo, sob situações normais de mercado.

\section{CONSIDERAÇÕES FINAIS}

O objetivo deste trabalho foi propor modelos de previsão para o volume exportado da fruticultura brasileira, com vistas a auxiliar no planejamento e controle de sua produção. Durante as análises, foi possível identificar nessas séries temporais a existência de tendências, sazonalidade, pontos influentes (atípicos) e heteroscedasticidade condicional na variância, bem como ter evidenciado o mecanismo causador de sua trajetória e o desempenho de tais modelos.

Nesse sentido, utilizou-se os modelos da família ARIMA/GARCH, cujos resultados foram considerados satisfatórios para seis das dez frutas analisadas, pertencentes ao grupo de alta qualidade de previsão. Contudo, fica a ressalva de que o uso de técnicas econométricas de previsão, sem estarem vinculadas a uma Revista Produção Online, Florianópolis, SC, v.15, n. 2, p. 553-572, abr./jun. 2015 
gestão do processo de previsão, dificilmente produzirão resultados satisfatórios que impactem positivamente no desempenho empresarial nos custos e na distribuição, haja vista a questionável performance dos modelos vista em quatro das dez frutas.

Ademais, os resultados sobre a heteroscedasticidade condicional via modelo IGARCH em duas frutas indicam a existência de uma quebra estrutural em sua variância, apontando para a existência de, pelo menos, dois regimes que a expliquem. A relevância desse achado é que tais fenômenos são comumente encontrados em séries históricas de preços, mas raramente aparecem vinculados a séries históricas de volume. E isso é comprovado pela escassez desse debate na literatura especializada nacional.

Para estudos futuros, além do uso do VaR no cálculo da perda máxima esperada ligada a um evento extremo em condições normais de mercado, também é recomendada a utilização de modelos econométricos que considerem a ocorrência de quebras estruturais (Markov Switching Models), investigando a sua validade tanto para as séries de dados que apresentaram o GARCH integrado como para as séries de média e baixa qualidade de previsão do volume, cujo desempenho pode estar associado à mudança de regime na série histórica, devido às crises econômicas ocorridas nos últimos 17 anos.

\section{REFERÊNCIAS}

BALCOMBE, K. The nature and determinants of volatility in agricultural prices: an empirical study from 1962-2008. Commodity Market Review 2009-2010, Rome, p.1-24, May, 2010.

BERA, A. K.; HIGGINS, M. L. ARCH models: properties, estimation and testing. Journal of Economic Surveys, v.7, n.4, p.305-366, Dec., 1993.

http://dx.doi.org/10.1111/j.14676419.1993.tb00170.x

BLIEMEL, F. Theil's forecast accuracy coefficient: a clarification. Journal of Marketing

Research, Chicago, v.10, p.444-446, 1973. http://dx.doi.org/10.2307/3149394

BOLLERSLEV, T. Generalized autoregressive conditional heteroskedasticity. Journal of Econometrics, v.31, n.3, p.307-327, Apr.1986.

http://dx.doi.org/10.1016/03044076(86)90063-1

BOX, G.E.P; JENKINS, G.M; REINSEL, G.C. Time series analysis: forecasting and control. 3. ed. New Jersey: Prentice Hall, 1994.

BRASIL. Ministério do Desenvolvimento, Indústria e Comércio Exterior. Sistema de Análise das Informações de Comércio Exterior (AliceWeb). 2014. Disponível em:

<http://aliceweb2.mdic.gov.br/>. Acesso em: 31/01/2014, 14:49. 
BREUSCH, T. S. Testing for autocorrelation in dynamic linear models. Australian Economic Papers, v.17, n. 31, p.334-355, Dec, 1978.

http://dx.doi.org/10.1111/j.14678454.1978.tb00635.x

BROOKS, C. Introductory econometrics for finance. 2. ed. New York: Cambridge University Press, 2008.

BUENO, G.; BACCARIN, J. G. Participação das principais frutas brasileiras no comércio internacional: 1997 a 2008. Revista Brasileira de Fruticultura, Jaboticabal, v.34, n.2, p.424-434, jun, 2012. http://dx.doi.org/10.1590/S0100-29452012000200015

DANESE, P.; KALCHSCHMIDT, M. The role of the forecasting process in improving forecast accuracy and operational performance. International Journal of Production Economics, v.131, n.1, p.204-214, May, 2011a. http://dx.doi.org/10.1016/j.ijpe.2010.09.006

The impact of forecasting on companies' performance: analysis in a multivariate setting. International Journal of Production Economics, v.133, n.1, p.458-469, Sep, 2011b. http://dx.doi.org/10.1016/j.ijpe.2010.04.016

DICKEY, D. A.; FULLER, W. A. Distributions of the estimators for autoregressive time series with a unit root. Journal of the American Statistical Association, v.74, n.366, p.427-431, jun., 1979. http://dx.doi.org/10.2307/2286348

ENGLE, R. F. Autoregressive conditional heteroscedasticity with estimates of the variance of United Kingdom inflation. Econometrica, v.50, n.4, p.987-1007, Jul, 1982.

http://dx.doi.org/10.2307/1912773

Estimates of the variance of U.S. inflation based upon the ARCH model. Journal of Money, Credit and Banking, v.15, n.3, p.286-301, Aug. 1983.

http://dx.doi.org/10.2307/1992480

ENGLE, R. F.; BOLLERSLEV, T. Modelling the persistence of conditional variances.

Econometric Reviews, v.5, n.1, p.1-50, 1986.

http://dx.doi.org/10.1080/07474938608800095

GODFREY, L. G. Testing against general autoregressive and moving average error models when the regressors include lagged dependent variables. Econometrica, v.46, n.6, p. 12931301, Nov, 1978. http://dx.doi.org/10.2307/1913829

HERSCOVICI, A. Historicidade, entropia e não-linearidade: algumas aplicações possíveis na Ciência Econômica. Revista de Economia Política, v.25, n.3, p.277-294, jul-set, 2005. http://dx.doi.org/10.1590/S0101-31572005000300007

IBRAF. Frutas Frescas. Exportação. Comparativo das exportações brasileiras de frutas frescas 2010-2009. São Paulo, 2011. Disponível em:

<http://www.ibraf.org.br/estatisticas/est_frutas.asp>. Acesso em: 06 set. 2011.

KALCHSCHMIDT, M. Best practices in demand forecasting: tests of universalistic, contingency and configurational theories. International Journal of Production Economics, v.140, n.2, p.782-793, Dec, 2012. http://dx.doi.org/10.1016/j.ijpe.2012.02.022

KWIATKOWSKI, D.; PHILLIPS, P. C. B.; SCHIMDT, P. SHIN, Y. Testing the null hypothesis of stationarity against the alternative of a unit root. Journal of Econometrics, v.54, n.1-3, p.159-178, Oct-Dec, 1992. http://dx.doi.org/10.1016/0304-4076(92)90104-Y 
LAMOUREUX, C. G.; LASTRAPES, W. D. Persistence in variance, structural change, and the GARCH model. Journal of Business \& Economic Statistics, v.8, n.2, p.225-234, Apr., 1990. http://dx.doi.org/10.1080/07350015.1990.10509794

LIMA, I. G.; CARMO, C. R. S.; MEGLIORINI, E. Preços na bananicultura: um estudo de variáveis com potencial de influenciar o preço da banana da região do Vale do Ribeira/SP. Revista de Auditoria, Governança e Contabilidade, v.1, n.1, p.16-36, 2012.

LIMA, J. R. F.; SILVA, J. S.; SANTOS, R. K. B. Comportamento dos preços da manga exportada do Brasil: 2004-2014. Organizações Rurais \& Agroindustriais, v. 15, n. 3, p. 370-380, 2013.

LJUNG, G. M.; BOX, G. E. P. On a measure of lack of fit in time series models. Biometrika, v.65, n.2, p. 297-303, Aug., 1978. http://dx.doi.org/10.2307/2335207

MAKRIDAKIS, S. Forecasting: its role and value for planning and strategy. International Journal of Forecasting, v.12, n.4, p.513-537, Dec., 1996.

http://dx.doi.org/10.1016/S0169-2070(96)00677-2

MORETTIN, P. A; TOLOI, C. M. C. Análise de séries temporais. 2. ed. São Paulo: Edgard Blücher, 2006.

MORETTIN, P. A. Econometria financeira: um curso em séries temporais financeiras. 2. ed. São Paulo: Edgard Blücher, 2011.

NZAKU, K.; HOUSTON, J. E.; FONSAH, E. G. Analysis of U.S. demand for fresh fruit and vegetable imports. Journal of Agribusiness, v.28, n.2, p.163-181, fall, 2010.

OLIVEIRA, A. M. B.; MELO SOBRINHO, M. J. V. Previsão do preço de venda da uva Itália e da manga Tommy produzidas no Vale do São Francisco via análise de séries temporais: um estudo de caso. Cadernos do IME - Série Estatística, v.27, p.29-43, 2009.

OLIVEIRA, A. M. B. Previsão do preço de venda de não-commodities agrícolas via análise de séries temporais: um estudo sobre a fruticultura comercializada no Vale do São Francisco. Revista Desenbahia, Salvador, n.13, p. 71-97, set. 2010.

PINDYCK, R.; RUBINFELD, D. Microeconomia. 8. ed. São Paulo: Pearson, 2013. SAID, E. S.; DICKEY, D. A. Testing for unit roots in autoregressive-moving average models of unknown order. Biometrika, v.71, n.3, p.599-607, Dec., 1984.

http://dx.doi.org/10.2307/2336570

SOUZA, A. E. C.; PINHEIRO, A. W. S.; SILVA, E. C. S.; REIS, K. A. Utilização do método Box-Jenkins (ARIMA) na previsão de demandas de um produto de uma empresa de beneficiamento de açaí. In: ENCONTRO NACIONAL DE ENGENHARIA DE PRODUÇÃO, 33, Salvador, Anais... Rio de Janeiro: ABEPRO, 2013.

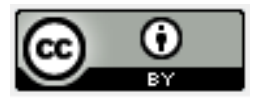

Artigo recebido em 01/12/2014 e aceito para publicação em 11/12/2014 DOI: http://dx.doi.org/ 10.14488/1676-1901.v15i2.1926 


\section{APÊNDICE A - Gráficos mensais do volume exportado por fruta}
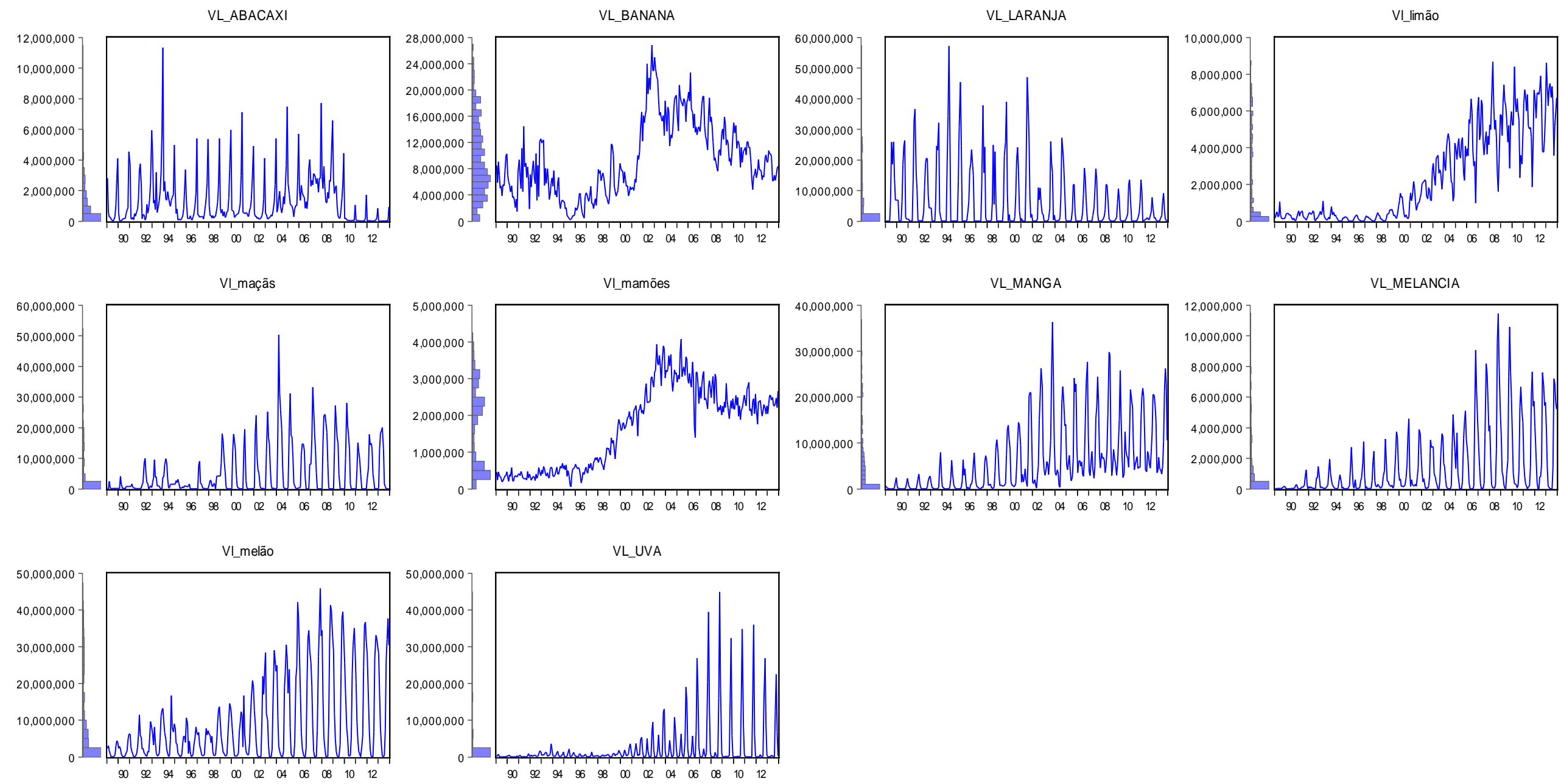

Fonte: Elaboração própria. 


\section{APÊNDICE B - Gráficos sazonais do volume exportado por fruta}

VOLUME $(\mathrm{kg})$ ABACAX

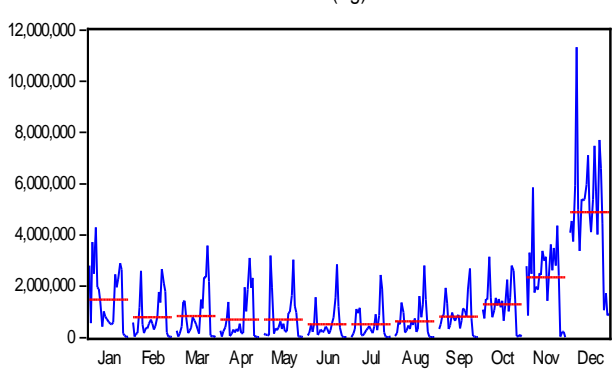

VOLUME $(\mathrm{kg})$ MAÇÃ

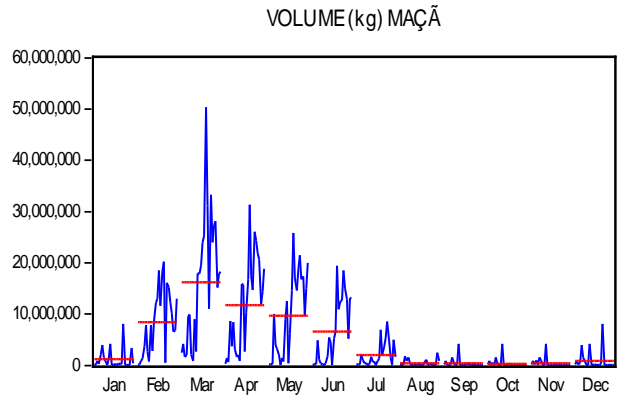

$\operatorname{VOLUME}(\mathrm{kg})$ MELÃO

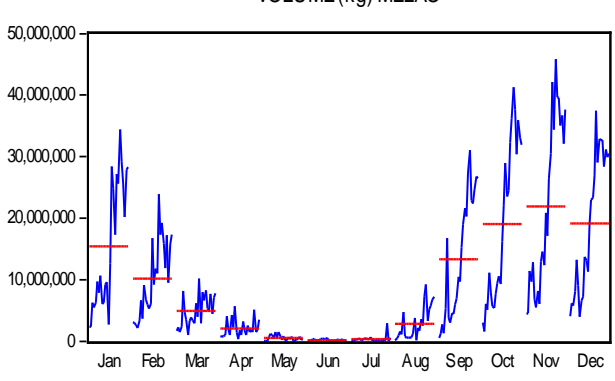

Fonte: Elaboração própria.
VOLUME $(\mathrm{kg})$ BANANA

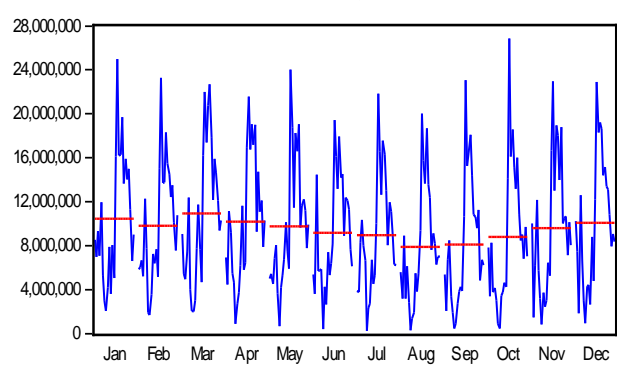

$\operatorname{VOLUME}(k g)$ MAMÃO

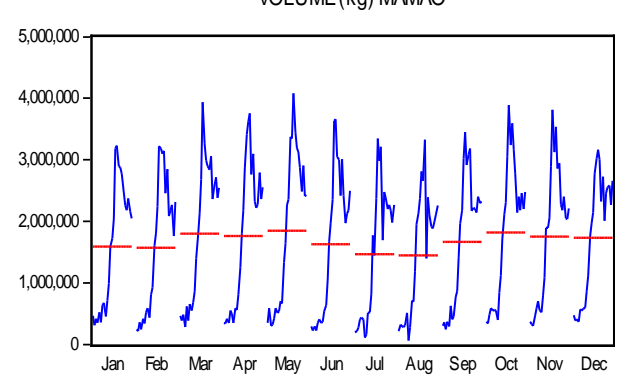

VOLUME $(\mathrm{kg})$ UVA

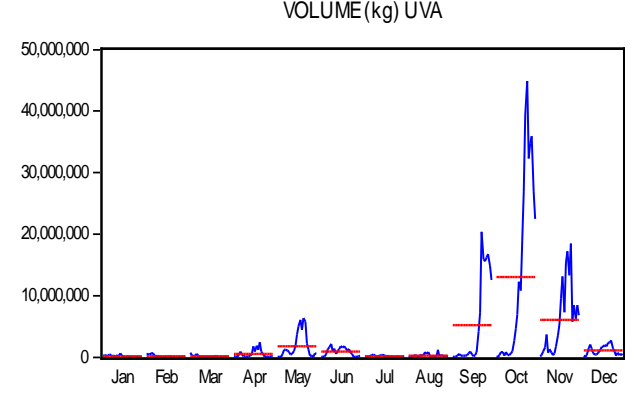

$\operatorname{VOLUME}(\mathrm{kg})$ LARANJA

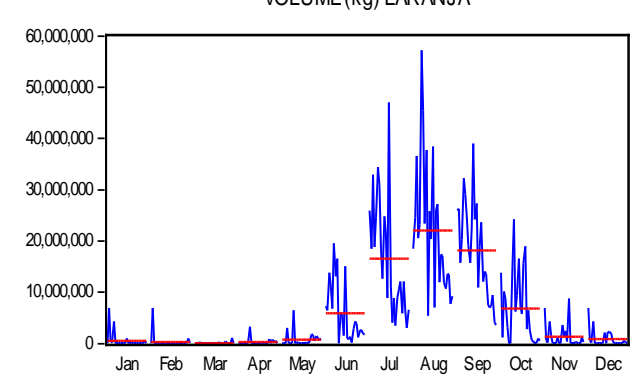

VOLUME $(\mathrm{kg})$ MANGA

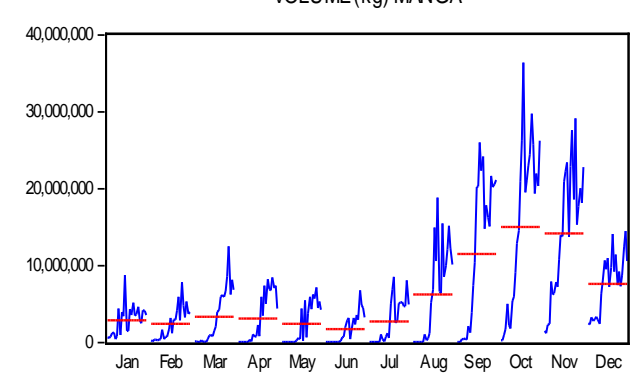

VOLUME $(\mathrm{kg})$ LIMÃOLIMA

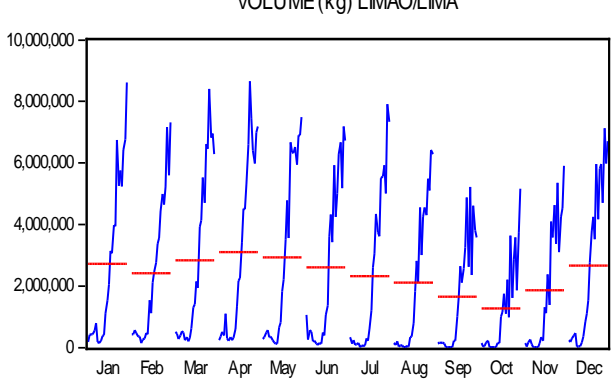

VOLUME(kg) MELANCIA

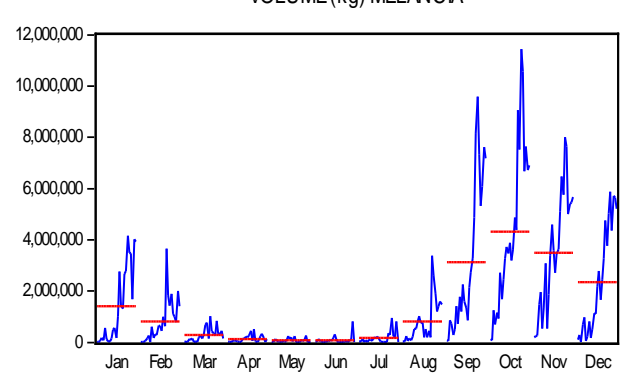

